\title{
CD1 Glycoprotein
}

National Cancer Institute

\section{Source}

National Cancer Institute. CD1 Glycoprotein. NCI Thesaurus. Code C114988.

A family of glycoproteins expressed on the surface of various human antigen-presenting cells. They are related to the class I MHC molecules, and are involved in the presentation of lipid antigens to T-cells. The family is separated into group 1, expressed on cells specialized for antigen presentation, and group 2, expressed on a wider variety of cells. 